\title{
Avaliação da influência da oxigenação e da qualidade do sedimento sobre a sobrevivência de Scolelepis chilensis (Spionidae: Polychaeta) da Baía de Guanabara, Rio de Janeiro
}

\author{
Marcelo Borges Rocha* \\ Evelyn Machado Silva \\ Nury Eunice Sánchez Riascos \\ Centro Federal de Educação Tecnológica Celso Suckow da Fonseca - CEFET/RJ. \\ Av. Maracanã, 229. Maracanã, Rio de Janeiro - RJ - 20271-110 \\ * Autor para correspondência \\ rochamarcelo36@yahoo.com.br
}

Submetido em 11/05/2013

Aceito para publicação em 25/09/2013

\section{Resumo}

O presente trabalho teve como objetivo avaliar a resistência do poliqueta Scolelepis chilensis a variações de oxigenação e sedimento a fim de obter dados para estudos de impactos ambientais. Os indivíduos foram coletados na Praia do Flamengo, Rio de Janeiro. Combinações favoráveis das características ambientais da Baía de Guanabara explicam a sobrevivência dos organismos, embora o intenso aporte de poluentes gere áreas fortemente degradadas. Dentre os grupos da fauna bentônica, os poliquetas se destacam, desempenhando um importante papel no funcionamento e na estrutura das comunidades aquáticas. Scolelepis chilensis apresenta ampla distribuição devido às suas características morfológicas que lhes permitem adaptar-se a diversos ambientes marinhos. Contudo, o presente trabalho mostra que a espécie apresenta alto índice de mortalidade em condições de hipoxia, significando dizer que essa espécie é mais sensível a ambientes com menos oxigênio. E, ainda, em situação de hipoxia associada a sedimento contaminado, o índice de mortalidade aumenta consideravelmente, mostrando-se a mesma ser sensível a ambientes com sedimento contaminado e com pouca oferta de oxigênio. Assim, o intenso e contínuo aporte de poluentes, poderá conduzir a um futuro déficit de oxigênio e a condições anaeróbicas extremas, os quais poderão ocasionar a destruição de diversos ecossistemas marinhos.

Palavras chave: Baía de Guanabara; Poluição; Polychaeta

\section{Abstract}

Influence of oxygenation and quality of sediment on the survival of Scolelepis chilensis (Spionidae: Polychaeta) in Guanabara Bay, Rio de Janeiro. This study aimed to evaluate the tolerance of the polychaete Scolelepis chilensis to variation in oxygenation and sediment to obtain data for environmental impact studies. Individuals were collected at Flamengo Beach, Rio de Janeiro. Favorable combinations of environmental characteristics of Guanabara Bay explain the survival of the organisms, although the introduction of pollutants produces heavily degraded areas. Among the groups of benthic fauna, polychaetes especially play an important role in the functioning and structure of aquatic communities. Scolelepis chilensis is widely distributed due to its morphological characteristics, which allow it to adapt to diverse marine environments. However, this study 
showed that this polychaete has a high mortality rate under hypoxic conditions, meaning that this species is more sensitive to environments with low oxygen. Furthermore, the mortality rate increases considerably in situations of hypoxia associated with contaminated sediment, indicating that this species is sensitive to environments with contaminated sediment and low oxygen supply. Thus, the intense and continuous input of pollutants could lead to a future deficit of oxygen and extreme anaerobic conditions, which may cause destruction of various marine ecosystems.

Key words: Guanabara Bay; Pollution; Polychaeta

\section{Introdução}

As atividades humanas representam, direta ou indiretamente, a principal causa de alterações da diversidade biológica marinha. A taxa de degradação dos ecossistemas marinhos é bastante expressiva, sendo assim, a conservação da biodiversidade deve ser prioridade para a humanidade (VASCONCELOS, 2002).

$\mathrm{O}$ enriquecimento orgânico das águas marinhas apresenta-se como um dos fatores mais importantes dentre os distúrbios ambientais abordados em estudos recentes. A variação orgânica em determinada área acarreta mudanças em toda a estrutura física, química e biológica do ambiente. Em função dessas atividades observa-se uma queda significativa na qualidade da água e consequentemente na biodiversidade aquática.

Nos últimos anos, a Baía de Guanabara se tornou um dos ambientes estuarinos mais degradados do país, recebendo carga intensa de poluição da região metropolitana do Rio de Janeiro e municipalidades vizinhas (MENICONI, 2007).

Segundo Santi (2008), a Baía de Guanabara é a mais proeminente baía brasileira. Apesar de sua importância histórica, econômica, cultural e científica, se encontra entre os sistemas costeiros mais degradados do país, devido às constantes alterações sofridas desde o século XIX. Tal processo de degradação assumiu uma escala mais drástica nas últimas décadas em função do pleno desenvolvimento urbano-industrial.

A fauna bentônica é a primeira a sofrer os impactos da poluição, pelo fato de seu habitat natural estar localizado no meio sedimentar, local ecologicamente frágil e que ao ser afetado, traz implicações a todos os diferentes estratos que constituem o ecossistema (SANTOS, 2009).
Diversos estudos constataram a importância das comunidades bentônicas na avaliação da qualidade ambiental, sendo indicadas como grupo ecológico de grande relevância para o monitoramento no meio aquático. Isto se deve ao fato de grande parte dos indivíduos que compõem estas comunidades apresentarem mobilidade limitada ou possuírem hábitos sedentários, tendo assim como única estratégia de sobrevivência à adaptação às condições ambientais que lhes são impostas.

Segundo Rozbaczylo et al. (2006), uma estreita relação entre os grupos funcionais tróficos e o tipo de sedimento permite que os organismos identificados possam ser usados como variáveis biológicas, atuando como bioindicadores na pesquisa e predição de interações funcionais. Bioindicadores são espécies, grupos de espécies ou comunidades biológicas cuja presença, quantidade e distribuição indicam a magnitude de impactos ambientais (CALLISTO; GONÇALVES, 2002). Um bom bioindicador deve ser facilmente identificado, permitir caracterizar o estado de um ecossistema e evidenciar as modificações naturais ou provocadas (AMARAL et al., 1998).

Nos últimos anos, tem aumentado o uso de poliquetas como indicadores de poluição marinha devido à sensibilidade que apresentam em relação às variações do meio e sua abundância em termos qualitativos e quantitativos, quando comparados a outros organismos da fauna bentônica. Muitos poliquetas são extremamente resistentes à poluição marinha, especialmente aquela causada por esgotos orgânicos. Por essa razão, são utilizados em muitos casos como indicadores de poluição (REISH, 1986).

Segundo Dean (2008), alguns poliquetas têm sido utilizados com mais frequência em estudos sobre o impacto da poluição orgânica no ambiente marinho, 
dentre eles destacam-se indivíduos das famílias Spionidae, Nereididae, Capitellidae, Pilargidae e Cirratulidae. Foi comprovado, a exemplo disto, que Perinereis nuntia e Neanthes japonica possuem capacidade de digerir matéria orgânica de água com esgoto. Nereis arenicoedentata foi utilizada em estudos de toxicologia citogenética e Tharyx longisetosa é indicadora de enriquecimento orgânico. E ainda, Heteromastus filiformis, Laeonereis acuta e Scolelepis squamata, Capitella capitata estão associadas a locais enriquecidos organicamente e impróprios para banho (AMARAL et al., 1998).

A habilidade das comunidades bentônicas em responder rapidamente a mudanças ambientais pode variar de acordo com o clima. Elias et al. (2005), em um estudo na costa de Mar Del Plata, na Argentina, encontraram como indicador de enriquecimento orgânico no verão o poliqueta Prionospio sp. Um ano antes, no inverno, Elias et al. (2004) relataram Caulleriella sp. como espécie indicadora do enriquecimento orgânico nesta mesma região. Desta forma, percebe-se que a diversidade de poliquetas é alterada de acordo com a estação do ano.

Pelo fato dos poliquetas serem um dos principais componentes das comunidades bentônicas, as mudanças ambientais refletem-se diretamente na ecologia desses organismos. Várias espécies de poliquetas, tais como indivíduos das famílias Lumbrineridae e Maldanidae (BELAN, 2003) e Terebellidae (OLSGARD et al., 2003) foram utilizados como indicadores de condições ambientais. Há uma dificuldade em encontrar uma espécie particular de poliqueta como um indicador de um ambiente bentônico degradado, cada região geográfica parece responder de maneira própria às condições ambientais, determinando sua espécie dominante.

Em um estudo mais antigo sobre os efeitos da poluição nas comunidades bentônicas em Oslofjord na Noruega, Mirza e Gray (1981) notaram uma diminuição na diversidade de espécies ao longo de um gradiente de áreas relativamente limpas a áreas altamente impactadas pela poluição orgânica. Encontraram que algumas espécies como C. capitata, Polydora spp. e Nereimyra punctata eram abundantes em locais poluídos e consequentemente designadas como indicadores de locais poluídos. Destas três espécies, C. capitata e Polydora spp. já tinham sido estudadas por Pearson e Rosenberg (1978) como indicadores de enriquecimento orgânico.

O uso destes organismos como bioindicadores é baseado em um princípio simples: submetidos a condições adversas, os organismos se adaptam ou morrem. Portanto, os organismos que vivem em um dado ecossistema estão adaptados às suas condições ambientais e por isso devem refletir o nível de preservação de condições naturais ou as alterações provocadas pela emissão de substâncias poluentes. Desta maneira, são muito utilizados em testes de toxicidade.

Os testes de toxicidade devem ser considerados como uma análise fundamental no controle da poluição hídrica, pois se fundamentam na utilização dos organismos vivos que são diretamente afetados pelos desequilíbrios que ocorrem nos ecossistemas, uma vez que as análises químicas apenas identificam e quantificam as substâncias presentes na água ou sedimento, mas não detectam os efeitos sobre a biota (ZAGATTO; BERTOLETTI, 2006).

Deste modo, a ecotoxicologia surge como ferramenta de monitoramento ambiental, baseando-se principalmente nas respostas de organismos individuais a estressores diversos. Portanto, é uma ciência com objetivo próprio de estudo, testando o fenômeno da intoxicação ambiental com todas as suas consequências, com finalidade e com método (AZEVEDO; CHASIN, 2003).

A espécie escolhida como organismo-teste para os bioensaios realizados neste estudo foi o poliqueta Scolelepis chilensis. Os principais critérios utilizados na escolha foram: a disponibilidade, a abundância, a facilidade de cultivo e o conhecimento da biologia da espécie. O gênero Scolelepis é um dos gêneros que apresenta maior número de espécies, ocorrendo principalmente em ambientes praiais em quase todo mundo (BOLÍVAR; LANA, 1986). Sua distribuição tem sido associada à quantidade de matéria orgânica nos sedimentos e também à presença de areia fina.

Os indivíduos desta espécie alimentam-se de partículas coletadas por um par de palpos. Tais partículas 
podem ser representadas por fragmentos de algas, matéria orgânica, animais planctônicos, entre outros (PARDO; AMARAL, 2004).

O presente estudo teve como objetivo avaliar a resistência de Scolelepis chilensis, a variações de oxigenação da água combinadas com dois tipos de sedimentos, um menos poluído (Praia do Flamengo/RJ) e um mais poluído (Enseada de Botafogo/RJ). A análise foi realizada a fim de se obter dados relevantes para estudos ambientais não só no local referente à coleta, como outros locais da costa brasileira.

\section{Materiais e Método}

As coletas foram realizadas na Praia do Flamengo (22 $55^{\prime} 24.40^{\prime}$ 'S 4310'06.23”'W), que possui aproximadamente $1 \mathrm{~km}$ de extensão, se estendendo por todo o Parque Brigadeiro Eduardo Gomes, Aterro do Flamengo, Rio de Janeiro. Esta praia está sujeita à influência de efluentes urbanos não tratados provenientes da Baía de Guanabara. Apesar da maioria das praias da Baía de Guanabara ser imprópria para o banho, a Praia do Flamengo é uma das poucas que, em algumas épocas do ano, apresenta balneabilidade que possibilita o banho.

Os espécimes foram coletados nos dias 3 e 4 de março de 2012, na zona entremáres, utilizando-se pegador de fundo, pás e peneiras com malha de 0,5 $\mathrm{mm}$. Também foram coletadas amostras de sedimento local, utilizando-se os mesmos materiais, e água, com a utilização de recipientes plásticos. Nestes mesmos dias foram coletadas amostras de sedimento da Enseada de Botafogo para serem utilizadas no experimento como sedimento mais impactado. A Enseada de Botafogo foi selecionada a partir de dados do Instituto Estadual do Ambiente (INEA) que mostram ser, entre as praias do Rio de Janeiro, a mais impactada pelos efluentes oriundos da Baía de Guanabara.

Após a coleta, os organismos foram acondicionados em recipientes plásticos e levados para o Laboratório de Polychaeta da Universidade Federal do Rio de Janeiro (UFRJ), onde foram identificados até o menor táxon possível com auxílio de microscópio estereoscópico ZEISS Stemi SV11. Após a identificação, os poliquetas foram mantidos em aquários, com aeração constante, contendo água e sedimento próprios do local de coleta por quinze dias para a aclimatação.

Durante o desenvolvimento do experimento foram conferidos duas vezes por dia os valores de oxigênio dissolvido de todos os aquários, utilizando o oxímetro Milwaukee Mn 600. Os dados de temperatura também foram aferidos com um medidor multi-parâmetros digital PHT-028. Ambos os dados foram conferidos a fim de se manter o controle da faixa de normalidade para hipoxia, normoxia e hiperoxia. A salinidade foi mantida estável a $30 \%$ e medida através do salinômetro-refractómetro (Hanna MR).

Os poliquetas foram organizados em aquários com dois tipos de sedimento: Sedimento natural e menos impactado (S1), referente à Praia do Flamengo, e sedimento impactado (S2), coletado na Enseada de Botafogo.

Com a finalidade de tirar a umidade e tornar o sedimento azoico, o material foi processado em forno microondas por 6 minutos na potência máxima. O mesmo procedimento foi utilizado com a água. Em todos os aquários a água do mar foi diluída em água destilada a fim de se obter a salinidade de $30 \%$.

Foram considerados três níveis de variação para o oxigênio: hiperoxia $(\mathrm{O} 1)$, normoxia $(\mathrm{O} 2)$ e hipoxia $(\mathrm{O} 3)$, sendo o controle dos níveis de variação realizado através de bombas de oxigênio e temporizadores. Utilizou-se 12 aquários para cada um dos três níveis de oxigênio, sendo 6 aquários com sedimento impactado e 6 com sedimento natural, contendo 6 poliquetas cada um, totalizando 216 Scolelepis chilensis para todo experimento.

O ensaio foi mantido por 36 dias submetido a um fotoperíodo artificial de $12 \mathrm{~h}$ usando lâmpadas de luz fria controladas com um temporizador, sendo os organismos alimentados uma vez por semana com comida comercial para peixe moída.

Ao final dos 36 dias de ensaio, a sobrevivência dos indivíduos foi quantificada e os dados obtidos foram tabulados em Excel e processados no ambiente $\mathrm{R}$ estatística. O modelo estatístico utilizado considerou dois fatores: qualidade do sedimento e oferta de oxigênio. A influência destes fatores sobre a sobrevivência dos 
organismos foi avaliada mediante a aplicação do Modelo Linear Generalizado com uma resposta Binomial.

\section{Resultados}

A partir da média dos valores de oxigênio conferidos durante os 36 dias de experimento, foi constatado que o mesmo apresentou-se dentro das faixas de normalidade para as três variáveis de oxigênio a que foi submetido: hipoxia (O1) 2,5 $\pm 1 \mathrm{mg} / \mathrm{L}$; normoxia (O2) 5,0 $\pm 1 \mathrm{mg} / \mathrm{L}$; e hiperoxia (O3) 7,0 $\pm 1 \mathrm{mg} / \mathrm{L}$. A temperatura também se manteve na faixa apropriada, sendo esta $25 \pm 2{ }^{\circ} \mathrm{C}$ para todos os aquários.

Utilizando-se o programa $\mathrm{R}$ estatística para a obtenção dos dados, obteve-se o resultado apresentado na Tabela 1.
Com base nos dados da Análise de Variância, pode-se inferir que, com $95 \%$ de confiança, Scolelepis chilensis depende do tipo de oferta de $\mathrm{O}_{2}$ para sua sobrevivência. O sedimento, embora tenha certo grau de influência, pelos resultados estatísticos, não apresentou influência significativa sobre a sobrevivência da espécie. Contudo, quando ocorre a interação entre sedimento e oxigênio, a resposta da sobrevivência é alterada.

Através dos resultados obtidos observa-se que tanto no sedimento natural quanto no contaminado as condições de hiperoxia e normoxia não apresentaram índices elevados de mortalidade de $S$. chilensis, porém em condições de hipoxia, os organismos apresentaram maior mortalidade, significando dizer que essa espécie é mais sensível a ambientes com menor oferta de oxigênio (Figura 1). E, ainda, quando a situação de hipoxia

TABELA 1: Resultados da Anova com relação à análise estatística da influência do oxigênio e do sedimento. F value distribuição F de Snedecor; $\operatorname{Pr}(>\mathrm{F})$ - Grau de significância (*).

\begin{tabular}{lcccc}
\multicolumn{1}{c}{ Grau de liberdade } & $\begin{array}{c}\text { Soma dos } \\
\text { quadrados }\end{array}$ & $\begin{array}{c}\text { Média dos } \\
\text { quadrados }\end{array}$ & F value & $\operatorname{Pr}(>\mathbf{F})$ \\
\hline Sedimento 1 & 0,26519 & 0,26519 & 3,2869 & 0,09491 \\
Oxigênio 2 & 0,84087 & 0,42043 & 5,2112 & $0,02350^{*}$ \\
Sedimento: oxigênio 2 & 1,03648 & 0,51824 & 6,4235 & $0,01269^{*}$ \\
Resíduos 12 & 0,96815 & 0,08068 & & \\
\hline
\end{tabular}

FIGURA 1: Influência do sedimento e do oxigênio na sobrevivência de Scolelepis chilensis. Em relação ao sedimento 1 (natural) e 2 (poluído). Em relação ao oxigênio, 1 equivale a hiperoxia, 2 a normoxia e 3 a hipoxia.

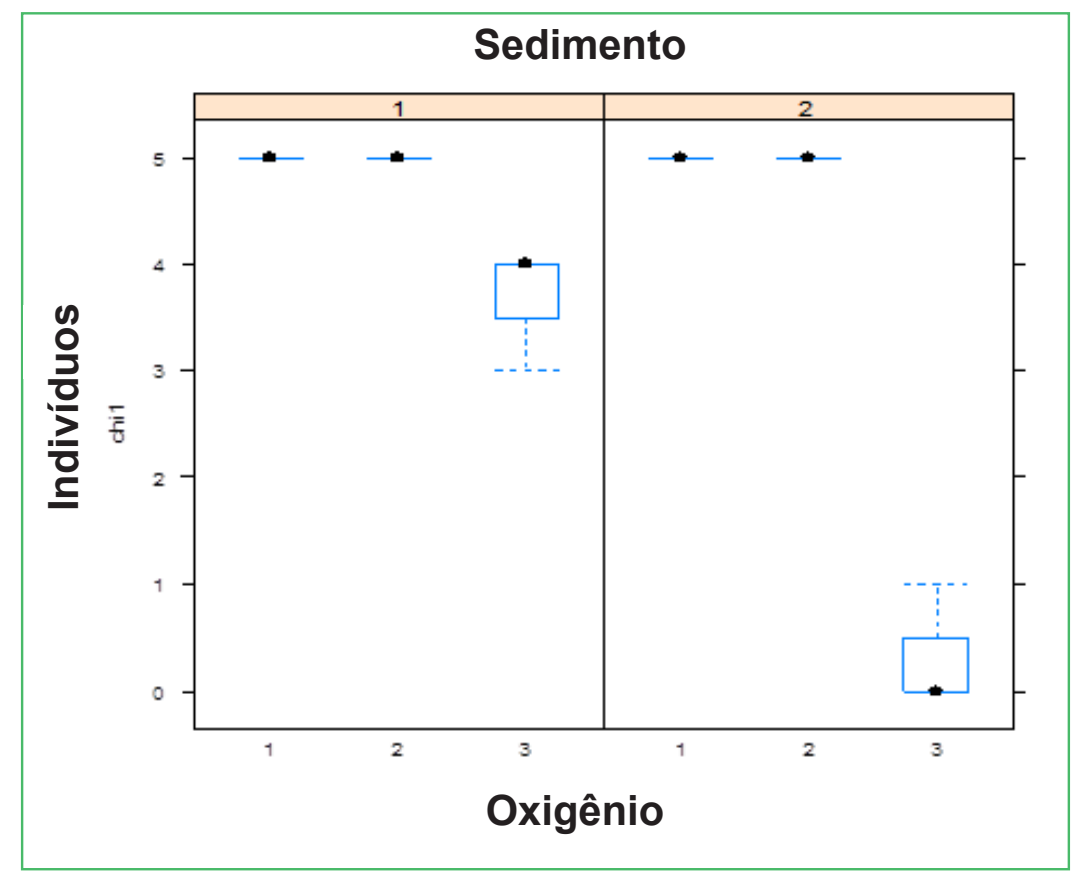


está associada ao sedimento contaminado, o índice de mortalidade aumenta consideravelmente, ou seja, essa espécie mostrou-se sensível a ambientes com sedimento contaminado e com pouco oxigênio.

\section{Discussão}

Os resultados obtidos permitiram atingir o principal objetivo proposto no trabalho, que foi avaliar a resistência de Scolelepis chilensis quando submetido a variações de oxigenação e sedimento.

Os resultados do presente trabalho evidenciaram a influência do aporte de diversos poluentes sobre a sobrevivência de organismos associados ao sedimento, podendo assim oferecer subsídio a futuros programas de monitoramento ambiental no local e em outros locais da costa brasileira. Tais resultados representam importantes contribuições na avaliação dos efeitos de atividades antrópicas sobre ecossistemas localizados próximos a grandes centros urbanos, uma vez que geram um panorama real sobre a qualidade ambiental.

Diversos autores têm estudado o efeito do déficit de oxigênio dissolvido sobre a comunidade bentônica que habita sedimentos arenosos. Rainer e Fitzhardinge (1981) descobriram que baixos níveis de oxigênio são um dos fatores mais importante na limitação de espécies em locais extremos quando comparados com outros fatores, como temperaturas e salinidades.

Rosenberg e Loo (1988) concluíram que períodos de deficiência de oxigênio podem afetar negativamente a abundância, distribuição e biomassa das espécies. Rosenberg et al. (1992) consideram a hipoxia como fator estruturante para comunidades marinhas bentônicas. Esses estudos vão ao encontro dos resultados obtidos neste trabalho, contribuindo para explicar a elevada taxa de mortalidade de $S$. chilensis quando submetidos à condições de hipoxia.

Segundo Desprez et al. (1992), baixas condições de oxigênio dissolvido nas águas de fundo contribuem para a redução significativa dos recursos vivos de muitos ambientes costeiros. As respostas da estrutura da comunidade bentônica à hipoxia dependem, principalmente, da intensidade e da duração da exposição do habitat à mesma (DIAZ; ROSENBERG, 1995). Um ambiente altamente impactado com um gradiente de oxigênio deprimido, mostra uma simplificação drástica das comunidades. A biomassa total é um bom fator de medição de estresse para os organismos da macroinfauna uma vez que os mesmos são produzidos somente em vias aeróbicas. Este descritor de estrutura da comunidade integra através do tempo a biodisponibilidade do oxigênio dissolvido da água para a biota (GONZALEZOREJA; SAIZ-SALINAS, 1998).

A deficiência de oxigênio aumenta ainda, potencialmente, a vulnerabilidade a predadores (KOLAR; RAHEL, 1993). Isto acontece porque o mecanismo que aumenta as chances de predação representa a resposta comportamental da infauna à hipoxia, com a diminuição da concentração de oxigênio no fundo, muitos organismos migram para a superfície dos sedimentos, tornando-se assim alvos fáceis para os predadores em densidades mais elevadas, do que as encontradas em períodos de normoxia (DIAZ et al.; 1992).

Inúmeros estudos articulam as variações observadas na estrutura da comunidade bentônica para as grandezas de medição que descrevem a natureza física e química do seu ambiente circundante. Os efeitos do enriquecimento orgânico, condições anóxicas ou alterações do fundo do mar por dragagem parecem seguir padrões bem definidos. Três grandes etapas são muitas vezes perceptíveis ao longo de gradientes espaciais: abundância e a diversidade de espécies aumentam, como táxons diferentes são capazes de sobreviver, e ocorre muitas vezes mudança da espécie dominante oportunista para espécies sensíveis.

Gonzalez-Oreja e Saiz-Salinas (1998), ao avaliarem a relação entre variáveis abióticas, sobretudo salinidade e oxigênio dissolvido e a estrutura da comunidade de poliquetas em um sistema estuarino na Espanha, observaram que a diminuição de oxigênio dissolvido causou um estresse bastante considerável nos organismos. Esses resultados corroboram os obtidos no presente estudo, onde se percebe que os indivíduos de S. chilensis mostraram-se mais sensíveis a situação de hipoxia em sedimento contaminado.

Lee et al. (2006), ao estudaram uma sequência temporal de colonização dos sedimentos de uma criação 
de peixes no Oceano Pacífico, observaram que os poliquetas Myriochele oculata, Pionosyllis heterocirrata (família Syllidae) e Euchone sp. (Família Maldanidae) encontradas no início do processo de acumulo de matéria orgânica foi diminuindo em quantidade e que $C$. capitata colonizou a região quando havia níveis intermediários de matéria orgânica. Por fim, constataram que $O$. adherens transformou-se em maioria numérica quando a matéria orgânica estava em níveis elevados, mesmo diminuindo os níveis de oxigênio. Desta forma, percebe-se que mais uma vez, o nível de oxigênio foi determinante para a resposta da comunidade bentônica.

Algumas espécies de poliquetas como, por exemplo, Capitella capitata podem servir como sinal de alerta para um ambiente marinho estressado ou como sinal de boa qualidade da água e do sedimento (DEAN, 2008).

Uma vez que as respostas esperadas de estudos utilizando biondicadores muitas vezes não podem ser instantâneas, dependendo da invulnerabilidade do ecossitema, torna-se fundamental avaliar as mudanças observadas. Os ensaios realizados não são uma tentativa de inferir preferências de habitat, nem aptidão para a espécie estudada. Na verdade, eles fornecem dados de base para explicar as dependências e interações presentes no ambiente aquático em questão, a fim de gerar subsídios relevantes para estudos ambientais.

Sendo assim, o presente trabalho contribuiu para o fornecimento de dados e informações relevantes para futuras avaliações de qualidade ambiental em ambientes sujeitos a influências antrópicas e alterações naturais, além de ser um documento útil para avaliações de balneabilidade e de interesse ecológico e econômico que a área de estudo oferece.

\section{Referências}

AMARAL, A. C.; MORGADO, E. H.; SALVADOR, L. B. Poliquetas bioindicadores de poluição orgânica em praias paulistas. Revista Brasileira de Biologia, São Carlos, v. 2, n. 58, p. 307-316, 1998.

AZEVEDO, F. A.; CHASIN, A. A. M. As bases toxicológicas da ecotoxicologia. São Carlos: Intertox, 2003. 340 p.

BELAN, T. A. Benthos abundance patterns and species composition in conditions of pollution in Amursky Bay (the Peter the Great Bay, the Sea of Japan). Marine Pollution Bulletin, Amsterdam, vol. 46, p. 1111-1119, 2003.

BOLIVAR, G. T. A.; LANA, P. C. Spionidae (Annelida: Polychaeta) do litoral do Estado do Paraná. Neritica, Curitiba v. 1, n. 3, p. 107148, 1986.

CALLISTO, M.; GONÇALVES, J. A vida nas águas das montanhas. Ciência Hoje, Rio de Janeiro, v. 31, n. 182, p. 68-71, 2002.

DEAN, H. K. The use of polychaetes (Annelida) as indicator species of marine pollution: a review. Revista de Biología Tropical, San José, v. 56, n. 4, p. 11-38, 2008.

DESPREZ, M.; RYBARCZYK, H.; WILSON, J. G.; DUCROTOY, J. P.; SUEUR, F.; OLIVES, R.; ELKAM, B. Biological impact of eutrophlcation in the Bay of Sorbonne and the induction and impact of anoxia. Netherlands Journal of Sea Research, Philadelphia, v. 3, n. 30, p.149-159, 1992.

DIAZ, R. J.; NEUBAUER, R. J.; SCHAFFNER, L. C.; PIHL, L.; BADEN, S. P. Continuous monitoring of dissolved oxygen in an estuary experiencing periodic hypoxia and the effect of hypoxia on macrobenthos and fish. Science of the Total Environment, Amsterdam, v. 2, n. 2, p. 1055-1068, 1992.

DIAZ, R. J.; ROSENBERG, R. Marine benthic hypoxia: review of ecological effects and behavioural responses on benthic macrofauna. In: BARNES, H; ANSELL, A. D.; GIBSON, R. N. (Ed.). Oceanography and Marine Biology Annual Review. Oxford: CRC Press, 1995. p. 245-303.

ELIAS, R.; PALACIOS, J. R.; RIVERO, M. S.; VALLARINO, E. A. Short-term responses to sewage discharge and storms of subtidal sand-bottom macrozoobenthic assemblages off Mar del Plata City, Argentina (SW Atlantic). Journal of Sea Research, Den Burg, v. 53, p. 231-242, 2005.

ELIAS, R.; VALLARINO, E. A.; SCAGLIOLA, M.; ISLA, F. I. Macrobenthic distribution patterns at a sewage disposal site in the inner shelf off Mar del Plata (SW Atlantic). Journal of Coastal Research, Fort Lauderdale, v. 20, p. 1176-1182, 2004.

GONZALEZ-OREJA, J. A.; SAIZ-SALINAS, J. I. Short-term spatio-temporal changes in urban pollution by means of faecal sterols analysis. Marine Pollution Bulletin, Amsterdam, v. 5, n. 8, p. 25-42, 1998.

KOLAR, C. S.; RAHEL, F. J. Interaction of a biotic factor (predator presence) and an abiotic factor (low oxygen) as an influence on benthic invertebrate communities. Oecologia, Heidelberg, v. 3, n. 95, p. 210-219, 1993.

LEE, H. W.; BAILEY-BROCK, J. H.; MCGURR; M. M. Temporal changes in the polychaete infaunal community surrounding a Hawaiian mariculture operation. Marine Ecology Progress Series, Oldendorf, v. 307, p. 175-185, 2006.

MENICONI, M. F. G. Hidrocarbonetos policíclicos aromáticos no meio ambiente: diferenciação de fontes em sedimentos e metabólitos em bile de peixes. 2007. 214 f. Tese (Doutorado em Química) Universidade Federal do Rio Grande do Norte, Natal. 2007.

MIRZA, F. B.; GRAY, J. S. The fauna of benthic sediments from the organically enriched Oslofjord, Norway. Journal of Experimental Marine Biology and Ecology, Amsterdam, v. 54, p. 181-207, 1981. OLSGARD, F.; BRATTEGARD, T.; HOLTHE, T. Polychaetes as surrogates for marine biodiversity: lower taxonomic resolution and indicator groups. Biodiversity Conservation, Madrid, v. 12, p. 1033-1049, 2003. 
PARDO, E. V.; AMARAL, A. C. Z. Feeding behavior of Scolelepis sp. (Polychaeta: Spionidae). Brazilian Journal of Oceanography, São Paulo, v. 52, n. 1, p. 75-79, 2004.

PEARSON, T. H.; ROSENBERG, R. Macrobenthic succession in relation to organic enrichment and pollution of the marine environment. Oceanography and Marine Biology, London, v. 16, p. 229-311, 1978.

RAINER, S. F.; FITZHARDINGE, R. C. Benthic communities in an estuary with periodic deoxygenation. Australian Journal of Marine \& Freshwater Research, Victoria, v. 32, n. 7, p. 227-243, 1981.

REISH, D. J. Benthic invertebrates as indicators of marine pollution: years of study. Marine Ecology Progress Series, Oldendorf, v. 63, n. 2, p. 163-175, 1986.

ROSENBERG, R.; LOO, L. O. Marine eutrophication induced oxygen deficiency: effects on soft bottom fauna, Western Sweden. Ophelia, Oxfordshire, v. 29, n. 5, p. 213-225, 1988.

ROSENBERG, R.; LOO, L. O.; MOLLER, P. Hypoxia, salinity and temperature as structuring factors for marine benthic communities in a eutrophic area. Netherlands Journal of Sea Research, Philadelphia, v. 30, n. 12, p. 121-129, 1992.
ROZBACZYLO, N.; MORENO, R.; DÍAZ, R. Poliquetos bentónicos submareales de fondos blandos de la región de Aysén, Chile: Ciados Amphinomida, Eunicida, Spionida, Sabellida y Scolecida (Anelida Polychaeta). Investigaciones Marinas, Valparaiso, v. 34, n. 1, p. 43-62, 2006.

SANTI, L. Estratégia reprodutiva e dinâmica populacional de Poecilochaetus australis Nonatu, 1963 (Polychaeta, Spionida) em local sujeito a influência de efluentes não tratados, Baía de Guanabara, Rio de Janeiro, Brasil: Projeto Australis. 2008. 198 f. Tese (Doutorado em Oceanografia) - Universidade de São Paulo, São Paulo. 2008.

SANTOS, J. M. N. Utilização de invertebrados bentônicos em ecotoxicologia sedimentar. 2009. 75 f. Dissertação (Mestrado em Biologia) - Universidade de Aveiro, Aveiro. 2009.

TOMMASI, L. R. Estudo de impacto ambiental. São Paulo: CETESB/TERRAGRAPH, 1994. 335 p.

VASCONCELOS, M. S. A condição humana e os oceanos. Breviário de Meditação. Lisboa: IPIMAR, 2002. 93 p.

ZAGATTO, P. A.; BERTOLETTI, E. Ecotoxicologia aquática: princípios e Aplicações. São Carlos: Editora Rima, 2006. 246 p. 